\title{
MACHINING CHARACTERISTICS OF THE HAVERSIAN AND PLEXIFORM COMPONENTS OF BOVINE CORTICAL BONE
}

\author{
Michael Conward \\ Graduate Research Assistant \\ conwam5@rpi.edu
}

\author{
Johnson Samuel * \\ Assistant Professor \\ samuei2@rpi.edu
}

\begin{abstract}
Rensselaer Polytechnic Institute, Department of Mechanical Aerospace and Nuclear Engineering, $1108^{\text {th }}$ Street, Troy, NY 12180, USA

* Corresponding Author
\end{abstract}

KEYWORDS: Bone microstructure; Haversian bone; Plexiform bone; Cutting; Microstructure-specific Machining

ABSTRACT: This paper investigates the characteristic differences observed while machining the haversian and plexiform components of a bovine cortical femoral bone. To this end micro-milling slotting experiments are performed on both the components by varying both the cutting velocity and the feed-per-tooth values. The scale of machining is chosen specifically to ensure sensitivity to the microstructural variations in the bone. The material properties of the microstructural components and their size-scale relative to the feed-per-tooth values are seen to dictate the failure mechanisms encountered during machining. The cutting force, surface roughness, and tool wear are all uniquely affected by the plexiform and haversian components of the cortical bone. In general, plexiform bone requires a higher cutting force than the haversian bone. While a higher cutting velocity can lower the surface roughness of haversian bone, it typically results in the most surface damage. The cutting force and surface roughness values for both the components show strain rate sensitivity. The tool wear is seen to be the highest while cutting parallel to the lamellar structures seen in the plexiform bone.

\section{INTRODUCTION}

It is well known that the microstructure of cortical bone and its material properties vary from patient to patient based on their age and health-history (McCalden et al., 1993; Rho et al., 2002). However, today's state-of-the-art bone surgeries perform routine machining procedures using cutting tools, tool-paths and processing conditions (i.e., feed-per-tooth and cutting velocities) that do not account for these microstructural/material property variations between patients (Barrera et al., 2008; Hofmann et al., 2011). This approach can result in damage to the cortical bone, which in turn contributes to an increase in patient recovery times (Hofmann et al., 2011; Kremers et al., 2013). In order to make the vision of patient-specific bone surgery protocols a reality, there is an imperative need to examine the machining responses of the microstructural constituents of cortical bone.

Bone machining studies have been conducted since the early 1970s, by both machining and biomedical researchers (Childs and Arola, 2011; Deglurkar et al., 2007; Jacobs et al., 1974; James et al., 2012; Sugita and Mitsuishi, 2009; Sugita et al., 2009). More recently, the experimental and mechanistic modeling work of (James et al., 2012) has been instrumental in shedding light on the cutting forces encountered during the machining of bovine cortical bone. While these studies have aided bone surgeries to-date, for the most part these were all macro-scale machining studies that were insensitive to microstructural variations in the bone. Furthermore, machining researchers have not paid enough attention to the bone microstructure while interpreting their results. For example, the cortical bovine bone is primarily made up of two load-carrying components viz., harvesian bone and plexiform bone, both of which have distinctly different microstructure and properties (Manilay et al., 2013). However, to-date the machining studies have not isolated the effects of these individual components on the machining responses of interest.

Biomedical researchers on the other hand have primarily focused on the clinical evaluation of surgical tools and therefore, their work has failed to provide any fundamental insight linking bone microstructure to its machining characteristics (Ortmaier et al., 2006; Park et al., 2010; Ueda et al., 2010). This state of the bone machining literature is in stark contrast to the publications in the area of the material behavior of bone that have focused heavily on the effect of age/bone-type related variations in the microstructure (McCalden et al., 1993; Rho et al., 2002).

The objective of this research is to investigate the characteristic differences observed while machining the haversian and plexiform components of a bovine cortical femoral bone. To this end, the haversian and plexiform bones are first characterized for their microstructure and nanoindentation properties. Micro-milling slotting experiments are then performed by varying both the cutting velocity and the feed-per-tooth values. The scale of machining is chosen specifically to ensure sensitivity to the microstructural variations. Finally, the machining results are interpreted in light of the material characterization work.

The remainder of this paper is divided into the following sections. Section 2 discusses the microstructure of the haversian and plexiform bones along with their nanoindentation properties followed by the details of the micro-milling experiment. Section 3 discusses the different failure mechanisms encountered in haversian and plexiform bones and then shows the trends observed in the cutting forces, surface roughness and tool wear. Finally, Section 4 presents the specific conclusions that can be inferred from this work. 


\section{MATERIAL AND METHODS}

This section will first discuss the specimen preparation and microstructure characterization efforts. This will be followed by the results from the nanoindentation study and a description of the micro-machining experimental setup.

\subsection{Specimen Preparation}

The bovine femoral bone used in this study was harvested from a 36 month old cow. The mid-body of the femoral bone shaft (i.e., mid-diaphysis) was sectioned using a Mar-Med ${ }^{\mathrm{TM}}$ bone saw, under constant water irrigation. As can be seen in Fig. 1, the circular cross-section of the femoral bone can be divided into four quadrants, viz., the anterior (A), posterior (P), lateral (L) and medial (M) quadrants. For a mature bovine bone, the anterior quadrant (A) is dominated by the plexiform bone (also known as fibrolamellar bone), whereas the posterior quadrant $(\mathrm{P})$ is dominated by the haversian bone. The medial and lateral quadrants are made up of transition regions between the two types. For the purposes of this study, the pure plexiform and haversian components of the bone were harvested from the anterior and posterior quadrants, respectively. After harvesting, the samples were stored in a freezer at $-20^{\circ} \mathrm{C}$ until their use for the nanoindentation and micro-machining studies. The samples were all thawed for an hour before conducting the tests following protocols of (Sugita and Mitsuishi, 2009).

\subsection{Microstructure Characterization}

Figure 2a depicts the characteristic cross-section of the haversian bone used in this study. As seen in Fig. 2a, the haversian bone is primarily composed of unidirectional fiber-like structures called osteons that are embedded in an interstitial matrix, with cement lines separating the two phases. The osteons themselves are made of concentric lamellae that contain pore-like structures called lacunae. They also have a central vascular channel called the harvesian canal that contains blood, lymph and nerve fibers. To characterize the size of these structures in the bone samples, a total of sixteen micrographs $(\sim 1950 \mu \mathrm{m} \mathrm{X} 1500 \mu \mathrm{m})$ taken over multiple specimens were analyzed. The size of the osteons were characterized as ellipses with an average major diameter of $190 \pm 50$ $\mu \mathrm{m}$ and minor diameter of $140 \pm 40 \mu \mathrm{m}$. The cement lines were seen to have an average thickness of $13 \pm 4.5 \mu \mathrm{m}$. The haversian canals had an average diameter of $41 \pm 22 \mu \mathrm{m}$. The length of the osteons was seen to be in the 3-5 mm range.

Figure $2 \mathrm{~b}$ depicts a characteristic micrograph of the plexiform bone. As seen, the plexiform bone contains alternating layers of woven and lamellar bone, giving it an appearance of a sandwich-structured composite. The woven/lamellar structures appear to be well-aligned within the plexiform bone. The regions of lamellar bone also show the presence of blood vessels. The woven and lamellar bones are seen to have average thicknesses of $70 \pm 20 \mu \mathrm{m}$ and $50 \pm 10 \mu \mathrm{m}$, respectively. The blood vessels themselves have an average diameter of $29 \pm 14 \mu \mathrm{m}$.

\subsection{Nanoindentation}

A Hysitron TI 900 TriboIndenter was used to perform nanoindentation tests on the haversian and plexiform bone samples used in this study. In order to prepare the samples for the nanoindentation tests, they were first polished using 600 and 1500 grit sandpaper, followed by a $5 \mu \mathrm{m}$ and $1 \mu \mathrm{m}$ alumina slurry on a wet polishing pad. Afterwards, the specimens were placed in an ulstrasonic bath for 15 minutes to remove the embedded alumina powder and surface debris, and then air dried at room temperature. For the haversian bone samples, a series of indents were carried out on the osteons, interstitial matrix and cement lines. Similarly, in the plexiform bone, both woven and lamellar components were tested. In order to prevent overlap of the residual plastic deformation zones, the spacing between consecutive indents was kept at 3-5 times the indent width.

For the nanoindentation tests, a Berkovich indenter was used to apply a $2000 \mu \mathrm{N}$ load-controlled indent using a trapezoidal function with a $5 \mathrm{sec}$. load, $5 \mathrm{sec}$. hold and $5 \mathrm{sec}$. unload cycle (Fig. 3). The $5 \mathrm{sec}$. hold period was used to allow for the stabilization of any creep in the samples. The elastic modulus was calculated from the slope of the upper portion of the unloading curve as per the protocols laid out by (Hoffler et al., 2000). The hardness value was measured by dividing the load by the area of contact of the indenter.

The elastic modulus and hardness results are summarized in Table 1. For the plexiform bone, the woven regions that make up $60 \%$ of its composition, were seen to have a significantly higher hardness and modulus value compared to the lamellar regions. This trend is attributed to differences in mineralization between the woven and the lamellar bone (Mayya et al., 2013) and has also been observed in past nanoindentation studies (Akhtar et al., 2011). No significant differences were observed between the properties measured for the osteons and the interstitial matrix. While this finding is similar to the nanoindentation cases seen in (Hoffler et al., 2000; Montalbano and Feng, 2011; Rho et al., 2002), it is different from the finding of (Budyn et al., 2012; Wang et al., 2006) who reported a higher modulus and hardness value for the interstitial matrix. These discrepancies in literature may be attributed to the anatomical location or variation in the diaphyseal region of the bone specimen (Hoffler et al., 2000). While these discrepancies remain unresolved, there is general acceptance of the notion that the overall mechanical integrity of the haversian bone is related to the area fraction of the osteons (Rho et al., 2002), which was estimated to be $~ 71 \%$ for the samples tested in this study.

For the haversian bone, the cement line was measured to have the lowest hardness and stiffness value when compared to the osteons and the interstitial matrix. This finding is in line with that of (Montalbano and Feng, 2011; Budyn and Hoc, 2010; Jonvaux et al., 2012) who reported the modulus and hardness values to be $~ 30 \%$ lower than the surrounding microstructures. However, recent work by (Malandrino et al., 2012) involving ultrasonic testing has revealed the possibility of nanoindentation-induced damage to be 
the likely reason for a lower elastic modulus measurement for the cement line. There is also poor agreement on the extent of mineralization of the cement line, which has been described both as a highly mineralized material (implying a brittle and stiff nature) and as a poorly mineralized material (Montalbano and Feng, 2011; Skedros et al., 2005). Modeling studies have shown that stiffer cement lines result in crack propagation around the osteons, whereas a lower modulus increases the chance of osteonal fracture (Li et al., 2013a). A combination of both these failure modes has been reported in experimental literature (Currey, 2012; Li et al., 2013b; Ritchie et al., 2005), indicating that the mechanical properties of cement lines likely vary with age and location (Li et al., 2013a).

\subsection{Machining Experiments}

The scale of machining chosen has to be such that it is sensitive to the microstructural variations seen in the cortical bone components. A micro-scale machining process such as micro-milling (Liu et al., 2004) is appropriate for such a study for two reasons. First, the size-scale of the tooling (diameters $<1 \mathrm{~mm}$ ), and the feed-per-tooth (FPT) conditions (typically in the 1 -20 $\mu \mathrm{m}$ range) used during micro-milling are likely to make it more sensitive to the variations in the microstructure of the bone. Furthermore, the FPT values encountered in micro-milling are also representative of the values encountered during the most commonly used bone-sawing procedures (James et al., 2012).

Figure 4 depicts the experimental setup used to perform the micro-milling experiments for this study. The tests were conducted on a three-axis hybrid micro-machining center (Mikrotools ${ }^{\mathrm{TM}}$ DT-110, Singapore). An electric NSK air bearing spindle with maximum speed of 80,000 RPM was used to perform the milling tests. The bone workpieces were mounted on a Kistler 9256C1 dynamometer, to measure the cutting forces. As seen in Fig. 2b, the plexiform bone has an inherent directionality associated with the orientation of its woven/lamellar structures. Therefore, for the plexiform bone, slots were machined at orientations that are both parallel $\left(0^{\circ}\right)$ and perpendicular $\left(90^{\circ}\right)$ to its woven/lamellar structures. These samples will be designated as plexiform- $0^{0}$ and plexiform$90^{\circ}$, respectively, for the remainder of this paper.

Slotting tests were performed on the haversian, plexiform $-0^{0}$, and plexiform- $90^{0}$ samples using a commercially available 800 $\mu \mathrm{m}$, single fluted, tungsten carbide endmill (Performance Micro Tool, ET-1-0312-S). The workpiece was in the form of rectangular specimens harvested from the cortical bone as shown in Fig. 1. The feed-per-tooth (FPT) was varied between 1-30 $\mu$ m for this study. For every FPT value, multiple slots of $300 \mu \mathrm{m}$ depth were machined in each of the three samples. To study the effect of strain-rate, the experiments were conducted at varying cutting velocities of $\sim 63 \mathrm{~m} / \mathrm{min}$ and $\sim 125 \mathrm{~m} / \mathrm{min}$. These cutting speeds were chosen based on the range of speeds used in previous bone cutting studies (Childs and Arola, 2011; Deglurkar et al., 2007; Jacobs et al., 1974; James et al., 2012; Sugita and Mitsuishi, 2009; Sugita et al., 2009). It should be noted that at the higher cutting speed of $125 \mathrm{~m} / \mathrm{min}$, tests could not be conducted at the FPT of $30 \mu \mathrm{m}$ given the limitation of the maximum allowable feed rate of the Mikrotools DT 110 machine tool. The cutting force data was collected by sampling at $30 \mathrm{kHz}$ using a high-speed data acquisition card (National Instruments). Table 2 outlines the cutting conditions used in this study.

\section{RESULTS AND DISCUSSION}

The following two issues become critical when studying the effect of bone microstructure on cutting outcomes. First, unlike carbon/glass fiber composites where the fibers are 5-6 $\mu \mathrm{m}$ in diameter (Calzada et al., 2010), here the critical microstructural features have a larger size-scale in the 10-200 $\mu \mathrm{m}$ range. This implies that for FPT values in the range of 1-30 $\mu \mathrm{m}$, used typically in surgery, the cutting edge can be expected to cut through individual microstructural features of the bone, for a significant duration of the cut. Second, the material properties of these microstructural features and their size-scale in relation to the FPT values will dictate the failure mechanisms encountered during machining. This section will highlight the differences in the failure mechanisms encountered for the haversian, plexiform $-0^{0}$ and plexiform $-90^{0}$ samples. For each of these three samples, a proposed failure mechanism will be first presented, based on their microstructural attributes. This will then be followed by evidence from the chip morphology data that backs up the proposed failure modes. Chip morphology data is only presented from the tests conducted at the cutting velocity of $125 \mathrm{~m} / \mathrm{min}$. The chips formed at the lower cutting speed of $63 \mathrm{~m} / \mathrm{min}$ are comparable in nature and are therefore not presented here for brevity.

\subsection{Haversian Bone}

As seen from the microstructural characterization (Fig. 2a), this bone consists of osteons that are held together by an interstitial matrix, with the cement line boundaries being $\sim 1 / 10^{\text {th }}$ the size of the osteons.

Figure 5a depicts a typical scenario that is encountered during the micro-milling of haversian bone. As identified in Fig. 5a, the tool cutting edge will distinctly see the change in the microstructural components, as it traverses along the path A-B-C-D-E-F, with A-B being the osteon, B-C being the haversian canal, C-D being the osteon, D-E being the cement lines, and E-F being the interstitial matrix. Variations of this heterogeneous pattern are expected to be seen for every single revolution of the cutting edge. While machining at low FPTs in the 1-5 $\mu \mathrm{m}$ range, ploughing is expected to be the primary mode of deformation due to the minimum chip thickness effect ( Liu et al., 2004). As a result, the failure would be dominated by the crushing-induced failure of the microstructural components. As the FPT is increased and cutting shifts to a shearing-dominated mode, the chips are likely to be more continuous. The surface of these chips is likely to show breaking patterns corresponding to failure happening at the haversian canals and cement lines.

The evidence supporting this proposed failure mechanism can be seen clearly in the chip morphology data presented in Figs.

5b-e. For the haversian bone, the chips formed at FPT of $1 \mu \mathrm{m}$ appear to be discontinuous, whereas they transition to more of a continuous chip at higher FPT values. The size of the chip debris at FPTs of $5 \mu \mathrm{m}$ and $10 \mu \mathrm{m}$ shows the presence of chip breakage at 
$\sim 80-100 \mu \mathrm{m}$, which corresponds to the osteonal radii. Evidence supporting inter-osteonal failure can also be seen in the surface morphology micrographs presented ahead in Section 3.4.2. This mode of failure during machining is not surprising given that it is also the predominant mode of failure reported in literature (Sugita and Mitsuishi, 2009; Sugita et al., 2009 ). As the FPT increases to $16 \mu \mathrm{m}$ and beyond, the chips are continuous in nature, indicating that a thicker chip is more likely to prevent the inter-osteonal cracks from propagating, thereby holding the chip together.

\subsection{Plexiform- $0^{0}$ Bone}

For the plexiform $-0^{0}$ case, the axis of the slot being machined is parallel to the woven/lamellar structures present in the plexiform bone. Figure 6a depicts the scenario that is likely to be encountered in this case where the woven bone (marked as $\mathrm{W}_{1}-\mathrm{W}_{9}$ ) is separated by the softer lamellar bone. At the beginning of every tool rotation, the cutting edge encounters the woven/lamellar structures at an orientation of $0^{0}$ (i.e., cutting velocity is parallel to the axis of the woven/lamellar structures). As the cutting edge rotates, this angle between the cutting velocity and the woven/lamellar structures changes continuously, taking on values of $90^{\circ}$ and $180^{\circ}$ at the center and the exit of the cut, respectively. However, this change in the angle is gradual over the revolution of the tool since both the woven and the lamellar structures have a significantly larger width of $\sim 50-70 \mu \mathrm{m}$, compared to the FPTs that are only in 1-30 $\mu \mathrm{m}$ range.

As identified in Fig. 6a, the tool cutting edge will distinctly see the microstructural components as it traverses along the path A-B-C-D, where A-B and C-D, represent the regions of the lamellar bone and B-C represents the region of the woven bone. The tool will see such alternating regions of woven and lamellar bone throughout the entire cut. Since the elastic modulus and the hardness of the lamellar bone is lower than that of the woven bone (Table 1), the machining will likely proceed through the cutting of the hard phase and the crack propagation at the lamellar boundary (i.e., inter-fibrolamellar failure). At low FPT values of 1-5 $\mu \mathrm{m}$, one is likely to see crushing of the woven/lamellar structures due to the minimum chip thickness effect ( Liu et al., 2004). At higher FPT values as one shifts to a shearing dominated regime, the chips are still expected to be discontinuous but now with width corresponding to the thickness of the woven/lamellar bone.

Figures 6b-e depict the chip morphology data for the plexiform- $0^{0}$ bone sample, which supports the proposed failure mechanisms. The chips formed at the FPT of $1 \mu \mathrm{m}$ show crushed fragments that are indicative of the ploughing effect caused at low FPTs. As the FPT values get higher, the size of the chips increases, however, the chips are never continuous. Instead, it shows characteristic chip breakage widths that are comparable to the thickness of the woven/lamellar bone (Fig. 6d-e).

\subsection{Plexiform- $90^{0}$ Bone}

The plexiform- $90^{0}$ scenario implies that the axis of the slot being machined is perpendicular to the axis of the woven/lamellar structures present in the plexiform bone. Figure 7 a depicts the scenario encountered while machining the plexiform- $90^{0}$ bone sample. At the beginning of the cut, the tool cutting edge encounters the woven/lamellar structures at an orientation of $90^{\circ}$ (i.e., cutting velocity is perpendicular to the axis of the woven/lamellar structures). This angle between the cutting velocity and the axis of the woven/lamellar structures changes as the tool rotates, taking values of $180^{\circ}$ and $270^{\circ}$ at the center and the exit of the cut, respectively.

As identified in Fig. 7a, the tool cutting edge will distinctly see the microstructural components as it traverses along the path A-B-C-D-E, where A-B, and C-D, represent the regions of cut in the woven bones $W_{2}-W_{3}$ marked in Fig. 7a, whereas B-C and D-E represent the regions of cut in the lamellar bone. At the maximum realized FPT condition encountered near the center of the slot, the tool is almost exclusively encountering a single woven/lamellar structure (region D-E). Given this situation, at low FPTs it is expected that one would still see the evidence of the minimum chip thickness effect in the form of crushing of the woven/lamellar bone. However, at higher FPT values, one is likely to see continuous chips as the tool edge would mostly be cutting only within a single woven/lamellar bone structure. Figures 7b-e depict the chip morphology data that supports the proposed failure mechanism. Continuous chips are seen even at a low FPT of $5 \mu \mathrm{m}$, which is different from the haversian and plexiform- $0^{0}$ case (Figs. 5 and 6 ).

\subsection{Other Machinability Measures}

This section presents the trends seen in the cutting forces, surface roughness and the tool-wear while machining the haversian, plexiform- $0^{0}$ and plexiform- $90^{\circ}$ bone samples.

\subsubsection{Cutting Forces}

Figures 8a-b depict the plot of the resultant peak-to-valley cutting forces, as a function of the FPT values for each of the three cutting scenarios. At a cutting velocity of $63 \mathrm{~m} / \mathrm{min}$, as the FPT is increased, the resultant cutting forces are seen to increase for all three types of bones (Fig.8a). At all FPT values the haversian bone is seen to have the lowest cutting force. This is likely because the occurrence of inter-osteonal failure aides the machining process (refer Section 2.3 and 3.1). The plexiform bone shows effects of directionality with the plexiform- $0^{0}$ sample having the highest cutting force, followed by the plexiform- $90^{0}$ sample. While machining at the $0^{0}$ orientation, the cutting edge encounters multiple woven and lamellar bone structures, in a single revolution (Fig. 6a). Given the alternate layers of the woven/lamellar bone, the cutting edge experiences a cyclic loading as it intermittently cuts through the harder woven bone regions while also promoting inter-fibrolamellar failure in the lamellar region. This cyclic loading of the tool is likely the reason for the increased cutting force encountered in this orientation. This is also supported by the work of (Kim et al., 2007) who showed that crack propagation across the lamellar bone takes more energy. While machining at the $90^{\circ}$ orientation, for a major portion of the tool revolution, the tool edge is cutting through a single woven/lamellar structure and not multiple woven/lamellar 
regions as encountered in the $0^{0}$ orientation. As a result there is no cyclic loading of the tool edge, which appears to result in a lower cutting force.

The cutting forces appear to show some strain-rate sensitivity as the cutting velocity is increased to $125 \mathrm{~m} / \mathrm{min}$ (Fig. 8b). For all the bone types, on average the cutting forces appear to be lower at higher cutting speeds at all FPTs. However, haversian bone still has the lowest cutting force. The directional differences between the plexiform- $0^{0}$ and plexiform- $90^{0}$ are seen to reduce at these higher velocities. The specific mechanisms for this behavior are unclear, and need to be further investigated using high-speed orthogonal machining studies.

In the cortical bone cutting studies to-date, the researchers have only reported average cutting forces without identifying the contributions by the specific microstructural components. The trends seen in the cutting forces reveal that on an average, the plexiform bone is the main contributor to the overall cutting force experienced during the machining of bovine cortical bone. In general, machining at the higher cutting velocity is seen to reduce the cutting force, however it could also lead to the possibility of necrosis (Childs and Arola, 2011) and should therefore be carefully selected. The result also points to the possibility of using different machining protocols while cutting through the plexiform and haversian bones.

\subsubsection{Surface Roughness}

Figures 9 a-f depicts representative surfaces that are seen at the cutting velocity of $63 \mathrm{~m} / \mathrm{min}$. For the haversian bone, as FPT is increased the feed marks become more visible on the surface. The surface is characterized mainly by the presence of cracks that are seen around the osteons, as indicated by the arrows in Figs. 9a,d. This supports the hypothesis that the failure is across the boundaries of the osteons. The extent of these sub-surface cracks in haversian bones is seen to increase with an increase in FPT (Figs. 9a, d).

The plexiform $-0^{0}$ and plexiform- $90^{0}$ surface profiles are similar to that of the haversian bone in that at lower FPT values the surface appears to be relatively smooth. While evidence of inter-fibrolamellar failure is visible at low FPTs (arrows in Figs. 9b,c), it is observed more clearly at higher FPTs in the form of cracks on the machined surface (Figs. 9e,f). The implications of these findings are that one could possibly create distinctly different types of machined surfaces as different constituents of the bovine cortical bone are being cut.

The surface roughness of the slot floor was measured for all bone types using the Zeta $20^{\mathrm{TM}}$ optical profilometer and the area surface roughness $\left(\mathrm{S}_{\mathrm{a}}\right)$ was calculated for the base of the slot. Figures 10a-b depict the plot of the average surface roughness (Sa), as a function of the FPT values for each of the three cutting scenarios. At the lower cutting velocity of $63 \mathrm{~m} / \mathrm{min}$ the surface roughness trends are seen to be opposite of the trends seen in the cutting force, in that the haversian bone has the highest surface roughness while the plexiform $-0^{0}$ bone has the lowest surface roughness. For the three bone types, the Sa value is seen to increase with FPT especially after a FPT of $10 \mu \mathrm{m}$.

At the higher cutting velocity of $125 \mathrm{~m} / \mathrm{min}$ and a FPT of $1 \mu \mathrm{m}$, it is again observed that haversian bone has the highest surface roughness and plexiform $-0^{0}$ has the lowest. However, as the FPT is increased the trends appear to reverse with the haversian bone surface roughness values decreasing. The surface roughness values for both plexiform- $0^{0}$ and plexiform- $90^{\circ}$ appear to be unaffected at higher velocities.

\subsubsection{Tool Wear}

In order to quantify the microstructural impact on tool wear, brand new single-fluted $800 \mu \mathrm{m}$ tools were first characterized under the Zeta $20^{\mathrm{TM}}$ optical profilometer. These were then used to perform three consecutive runs of half-immersion climb-milling cuts on each of the materials. Each run consisted of forty $5 \mathrm{~mm}$ long half immersion cuts. The axial depth-of-cut, FPT and cutting speed values were maintained at $100 \mu \mathrm{m}, 5 \mu \mathrm{m}$, and $63 \mathrm{~m} / \mathrm{min}$, respectively, for the duration of each of the runs. At the end of each run, the tool tip was imaged under the Zeta $20^{\mathrm{TM}}$ optical profilometer to record the extent of wear. Figures 11 a-d capture the progressive wear seen by the tool edge in the case of a plexiform $-0^{0}$ bone sample. Similar images were taken for all samples and the tool wear was analyzed using a digital image processing technique developed by (Arora et al., 2013).

Figure 11e depicts the trends seen in the tool wear for all the bone types, at the end of each run. It can be seen that at the end of the first two runs, haversian bone causes the most amount of wear followed by plexiform- $0^{0}$ and lastly plexiform- $90^{\circ}$. However, by the end of the third run, as the volume of material being machined increases, it can be observed that the plexiform- $0^{0}$ bone now has the highest wear while plexiform- $90^{\circ}$ still has the least wear.

As stated previously, when machining the plexiform- $0^{0}$ bone the tool is repeatedly cutting through multiple woven and lamellar regions. It is expected that with the woven bone having the highest hardness value and the cutting edge constantly having to reengage this hard material after cutting through its softer lamellar boundaries, this bone type would result in the most wear. Again, in the case of the haversian bone, the tool is repeatedly cutting through different material phases. However the difference in hardness between the osteon and interstitial matrix is not as drastic (Table 1). Therefore, a more linear increase in wear is observed in that case. When machining plexiform $-90^{\circ}$ the tool does not see as much of a material heterogeneity (refer Section 3.3), which can explain its lowest wear.

\section{CONCLUSIONS}

The following conclusions can be drawn from this work 
1. The overall machining response of cortical bone is composed of distinctly different contributions from both its haversian and plexiform components. The material properties of the microstructural constituents and their size-scale relative to the feed-pertooth (FPT) values dictate the failure mechanisms encountered during machining.

2. Haversian bone failure is primarily driven by inter-osteonal failure as indicated by the chip breakage size and the sub-surface cracks seen on the floor of the slots. For the plexiform $-0^{0}$ sample, failure is seen to proceed through the cutting of the hard woven bone and crack propagation at the lamellar boundary (i.e., inter-fibrolamellar failure). In the case of the plexiform- $90^{\circ}$ sample, the cutting edge does not see as much of an heterogeneity during the cut, thereby resulting in continuous chips even at the lowest FPT of $5 \mu \mathrm{m}$.

3. The micro-milling tests reveal that the cutting force, surface roughness, and tool wear are all uniquely affected by the plexiform and haversian components of the cortical bone. In general, plexiform bone requires a higher cutting force than haversian bone and while a higher cutting velocity can lower the surface roughness of haversian bone, it typically results in the most surface damage. Both the cutting forces and surface roughness values show strain rate sensitivity. Plexiform- $0^{0}$ sample results in the maximum tool wear.

\section{Acknowledgement}

The authors acknowledge funding support from the United States National Science Foundation CAREER award (CMMI 13-51275). The Rensselaer Center for Materials, Devices and Integrated Systems (cMDIS) is also acknowledged for the use of their imaging facilities.

\section{References}

Akhtar, R., Daymond, M.R., Almer, J.D., Mummery, P.M., 2011. Load transfer in bovine plexiform bone determined by synchrotron x-ray diffraction. J. Mater. Res. 23, 543-550. doi:10.1557/JMR.2008.0068

Arora, I., Samuel, J., Koratkar, N., 2013. Experimental investigation of the machinability of epoxy reinforced with graphene platelets. J. Manuf. Sci. Eng. 135, 41007.

Barrera, O.A., Haider, H., Garvin, K.L., 2008. Towards a standard in assessment of bone cutting for total knee replacement. Proc. Inst. Mech. Eng. Part H J. Eng. Med. 222, 63-74.

Budyn, E., Jonvaux, J., Funfschilling, C., Hoc, T., 2012. Bovine Cortical Bone Stiffness and Local Strain are Affected by Mineralization and Morphology. J. Appl. Mech. 79, 011008. doi:10.1115/1.4004644

Budyn, E., Hoc, T., 2010. Analysis of micro fracture in human Haversian cortical bone under transverse tension using extended physical imaging. Int. J. Numer. Methods Eng. 82, 940-965.

Calzada, K.A., Samuel, J., Kapoor, S.G., DeVor, R.E., Srivastava, A.K., Iverson, J., 2010. Failure Mechanisms Encountered in MicroMilling of Aligned Carbon Fiber Reinforced Polymers. Trans. NAMRI/SME 38, 221-228.

Childs, T.H.C., Arola, D., 2011. Machining of Cortical Bone: Simulations of Chip Formation Mechanics Using Metal Machining Models. Mach. Sci. Technol. 15, 206-230. doi:10.1080/10910344.2011.580699

Currey, J.D., 2012. The structure and mechanics of bone. J. Mater. Sci. 47, 41-54.

Deglurkar, M., Davy, D.T., Stewart, M., Goldberg, V.M., Welter, J.F., 2007. Evaluation of machining methods for trabecular metal implants in a rabbit intramedullary osseointegration model. J. Biomed. Mater. Res. Part B Appl. Biomater. 80, 528-540.

Hoffler, C.E., Moore, K.E., Kozloff, K., Zysset, P.K., Brown, M.B., Goldstein, S.A., 2000. Heterogeneity of bone lamellar-level elastic moduli. Bone 26, 603-609.

Hofmann, S., Seitlinger, G., Djahani, O., Pietsch, M., 2011. The painful knee after TKA: a diagnostic algorithm for failure analysis. Knee Surgery, Sport. Traumatol. Arthrosc. 19, 1442-1452.

Jacobs, C.H., Pope, M.H., Berry, J.T., Hoaglund, F., 1974. A study of the bone machining process—orthogonal cutting. J. Biomech. 7, 131-136.

James, T.P., Pearlman, J.J., Saigal, A., 2012. Rounded cutting edge model for the prediction of bone sawing forces. J. Biomech. Eng. 134, 1-11. doi:10.1115/1.4006972

Jonvaux, J., Hoc, T., Budyn, É., 2012. Analysis of micro fracture in human Haversian cortical bone under compression. Int. j. numer. method. biomed. eng. 28, 974-998.

Kim, J., Niinomi, M., Akahori, T., Toda, H., 2007. Fatigue properties of bovine compact bones that have different microstructures. Int. J. Fatigue 29, 1039-1050. doi:10.1016/j.ijfatigue.2006.09.018 
Kremers, H.M., Visscher, S.L., Moriarty, J.P., Reinalda, M.S., Kremers, W.K., Naessens, J.M., Lewallen, D.G., 2013. Determinants of direct medical costs in primary and revision total knee arthroplasty. Clin. Orthop. Relat. Res. 471, 206-214.

Li, S., Abdel-Wahab, A., Demirci, E., Silberschmidt, V. V, 2013a. Fracture process in cortical bone: X-FEM analysis of microstructured models. Int. J. Fract. 184, 43-55.

Li, S., Abdel-Wahab, A., Silberschmidt, V. V, 2013b. Analysis of fracture processes in cortical bone tissue. Eng. Fract. Mech. 110, 448-458.

Liu, X., DeVor, R.E., Kapoor, S.G., Ehmann, K.F., 2004. The mechanics of machining at the microscale: assessment of the current state of the science. Trans. ASME J. Manuf. Sci. Eng. 126, 666-678.

Malandrino, A., Fritsch, A., Lahayne, O., Kropik, K., Redl, H., Noailly, J., Lacroix, D., Hellmich, C., 2012. Anisotropic tissue elasticity in human lumbar vertebra , by means of a coupled ultrasound-micromechanics approach. Mater. Lett. 78, 154-158. doi:10.1016/j.matlet.2012.03.052

Manilay, Z., Novitskaya, E., Sadovnikov, E., McKittrick, J., 2013. A comparative study of young and mature bovine cortical bone. Acta Biomater. 9, 5280-8. doi:10.1016/j.actbio.2012.08.040

Mayya, A., Banerjee, A., Rajesh, R., 2013. Mammalian cortical bone in tension is non-Haversian. Sci. Rep. 3.

McCalden, R.W., McGeough, J.A., Barker, M.B., Court-Brown, C.M., 1993. Age-related changes in the tensile properties of cortical bone. The relative importance of changes in porosity, mineralization, and microstructure. J. Bone Jt. Surg. 75, 1193-1205.

Montalbano, T., Feng, G., 2011. Nanoindentation characterization of the cement lines in ovine and bovine femurs. J. Mater. Res. 26, 1036-1041.

Ortmaier, T., Weiss, H., Döbele, S., Schreiber, U., 2006. Experiments on robot assisted navigated drilling and milling of bones for pedicle screw placement. Int. J. Med. Robot. Comput. Assist. Surg. 2, 350-363.

Park, S.-Y., Shin, S.-Y., Yang, S.-M., Kye, S.-B., 2010. Effect of implant drill design on the particle size of the bone collected during osteotomy. Int. J. Oral Maxillofac. Surg. 39, 1007-1011.

Rho, J.Y., Zioupos, P., Currey, J.D., Pharr, G.M., 2002. Microstructural elasticity and regional heterogeneity in human femoral bone of various ages examined by nano-indentation 35, 189-198.

Ritchie, R.O., Kinney, J.H., Kruzic, J.J., Nalla, R.K., 2005. A fracture mechanics and mechanistic approach to the failure of cortical bone. Fatigue Fract. Eng. Mater. Struct. 28, 345-371.

Skedros, J.G., Holmes, J.L., Vajda, E.G., Bloebaum, R.O.Y.D., 2005. Cement Lines of Secondary Osteons in Human Bone Are Not Mineral-Deficient : New Data in a Historical Perspective 803, 781-803. doi:10.1002/ar.a.20214

Sugita, N., Mitsuishi, M., 2009. Specifications for machining the bovine cortical bone in relation to its microstructure. J. Biomech. 42, 2826-2829.

Sugita, N., Osa, T., Aoki, R., Mitsuishi, M., 2009. A new cutting method for bone based on its crack propagation characteristics. CIRP Ann. - Manuf. Technol. 58, 113-118. doi:10.1016/j.cirp.2009.03.057

Ueda, T., Wada, A., Hasegawa, K.-I., Endo, Y., Takikawa, Y., Hasegawa, T., Hara, T., 2010. Design optimization of surgical drills using the Taguchi method. J. Biomech. Sci. Eng. 5, 603-614.

Wang, X.J., Chen, X.B., Hodgson, P.D., Wen, C.E., 2006. Elastic modulus and hardness of cortical and trabecular bovine bone measured by nanoindentation. Trans. Nonferrous Met. Soc. China 16, Supple, s744-s748. doi:http://dx.doi.org/10.1016/S10036326(06)60293-8. 
Figure 1 - Single column

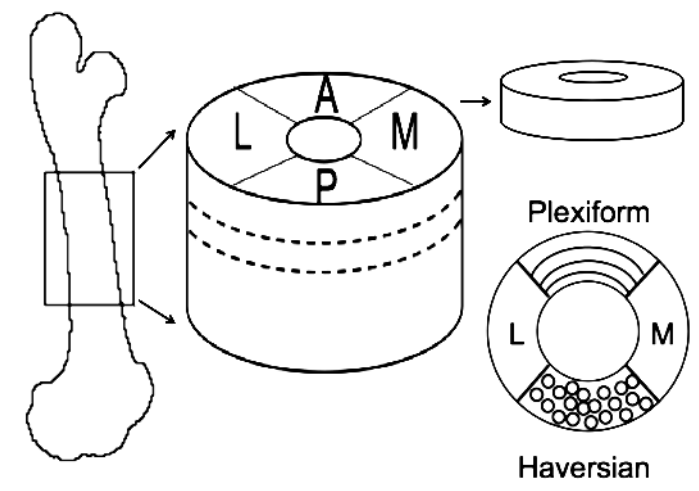

Figure 1: Schematic illustration of specimen preparation process 
Figure 2 - Single column

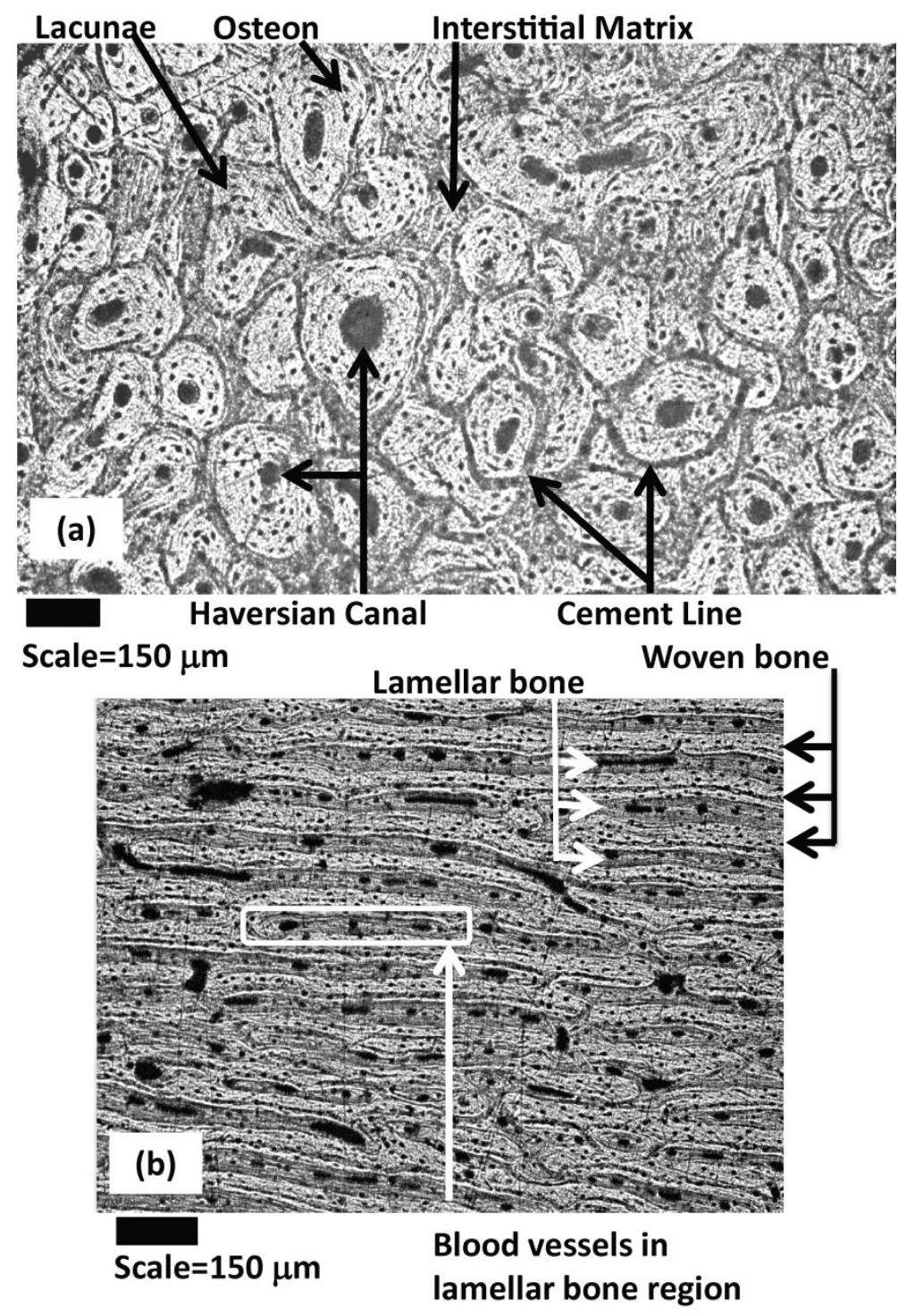

Figure 2: Microstructure characterization (a) micrograph of haversian bone (b) micrograph of plexiform bone 
Figure 3- Single column

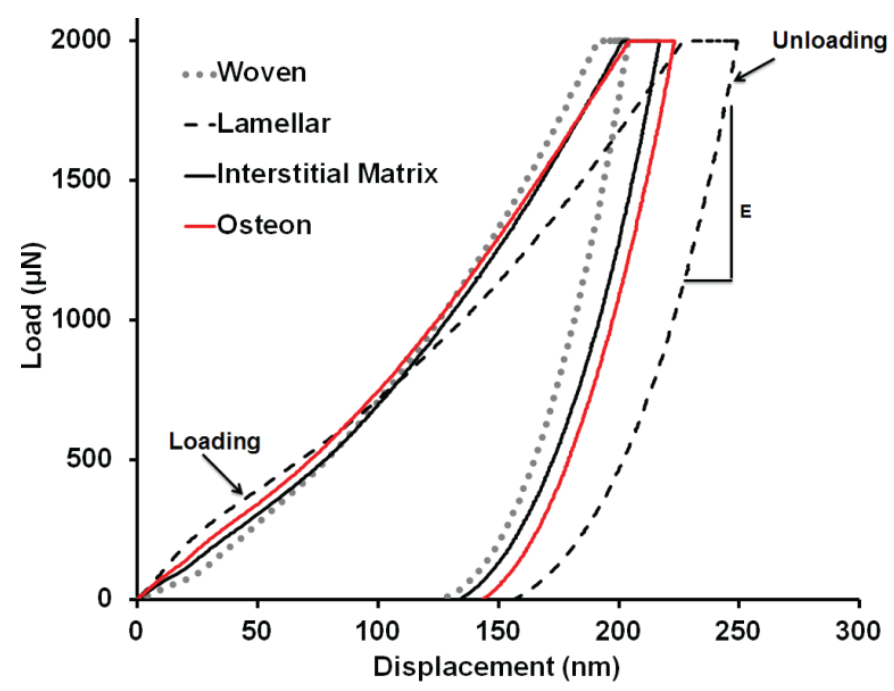

Figure 3: Nanoindentation load-displacement curve 
Figure 4- Single column

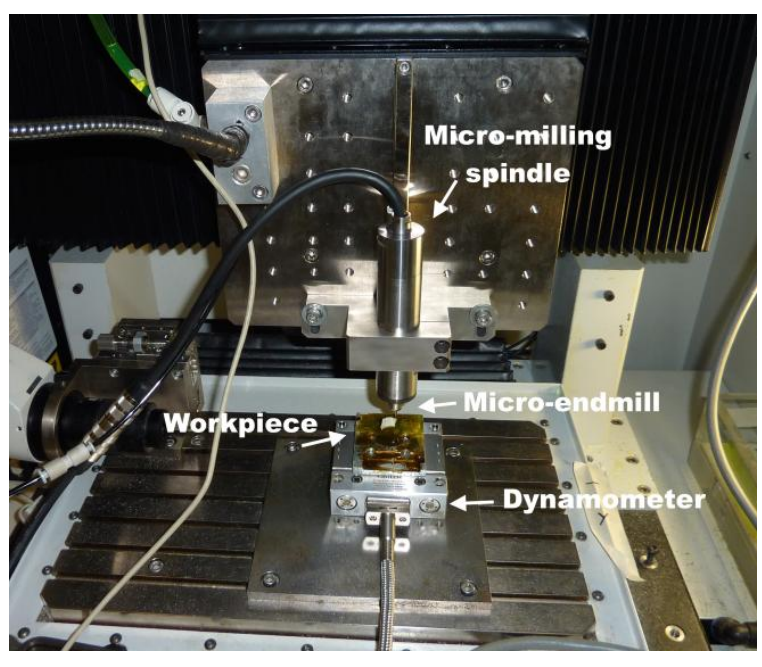

Figure 4: Micro-milling setup 
Figure 5- Single Column

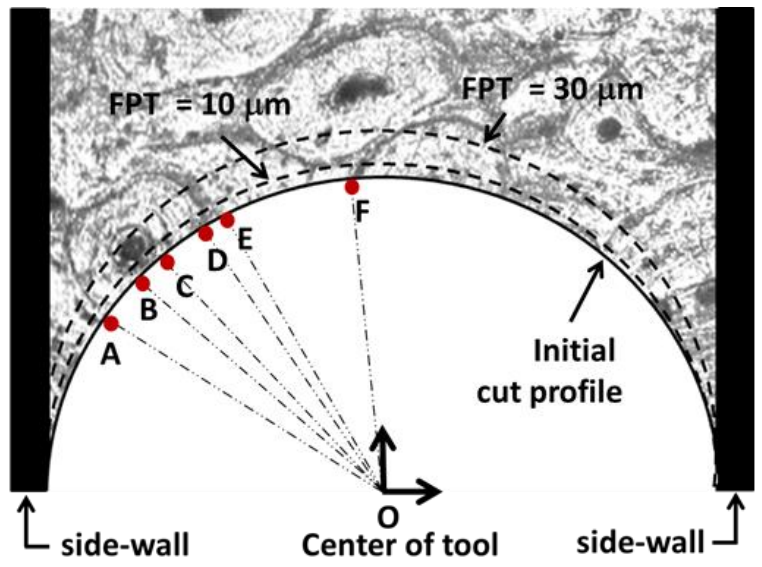

(a)
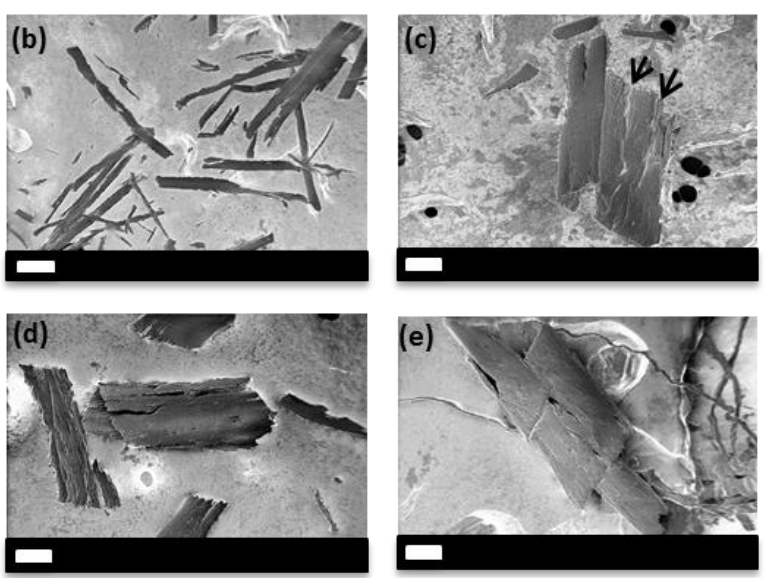

Figure 5: Failure mechanisms in haversian bone (a) heterogenity experienced by the cutting edge; (b)-(e) chip morphology at cutting velocity of $125 \mathrm{~m} / \mathrm{min}$ and FPTs of 1 $\mu \mathrm{m}, 5 \mu \mathrm{m}, 10 \mu \mathrm{m}$ and $16 \mu \mathrm{m}$, respectively. [Note: chip breakage in $(\mathrm{c}-\mathrm{d})$ points to inter-osteonal failure] (Scale = $100 \mu \mathrm{m})$ 
Figure 6- Single Column

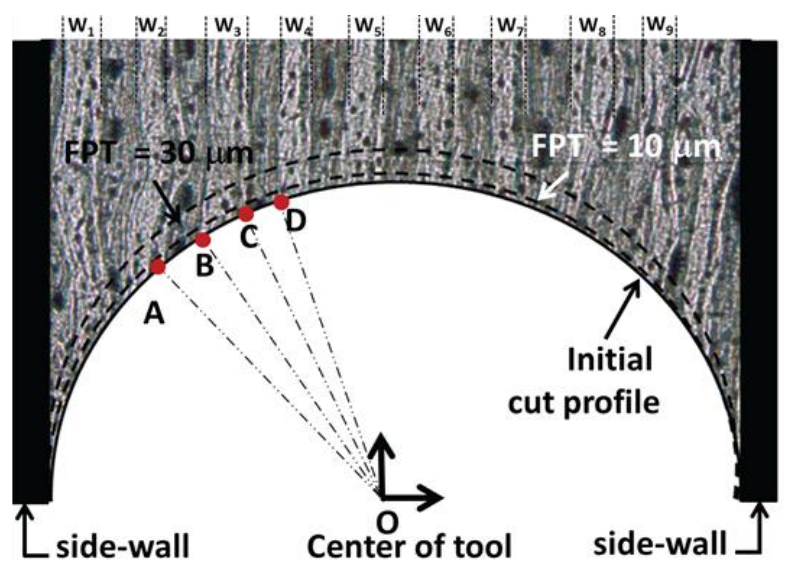

(a)
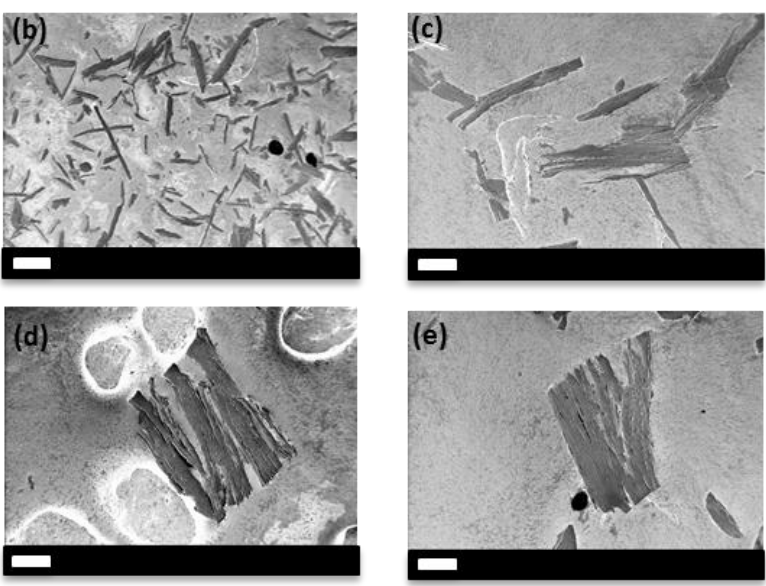

Figure 6: Failure mechanisms in plexiform $-0^{0}$ bone (a) heterogenity experienced by the cutting edge; (b)-(e) chip morphology at cutting velocity of $125 \mathrm{~m} / \mathrm{min}$ and FPTs of 1 $\mu \mathrm{m}, 5 \mu \mathrm{m}, 10 \mu \mathrm{m}$ and $16 \mu \mathrm{m}$, respectively. [Note: chip breakage in (d-e) points to inter-fibrolamellar failure] (Scale $=100 \mu \mathrm{m}$ ) 
Figure 7- Single Column

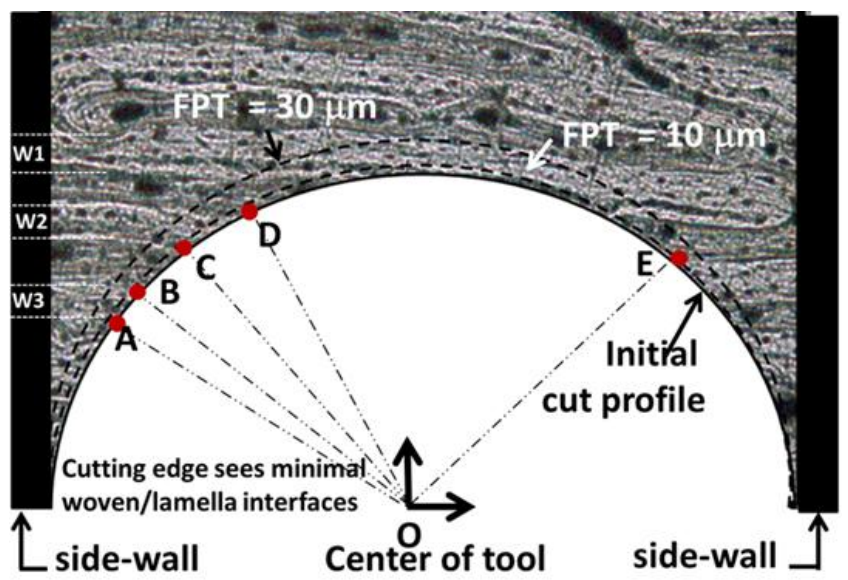

(a)
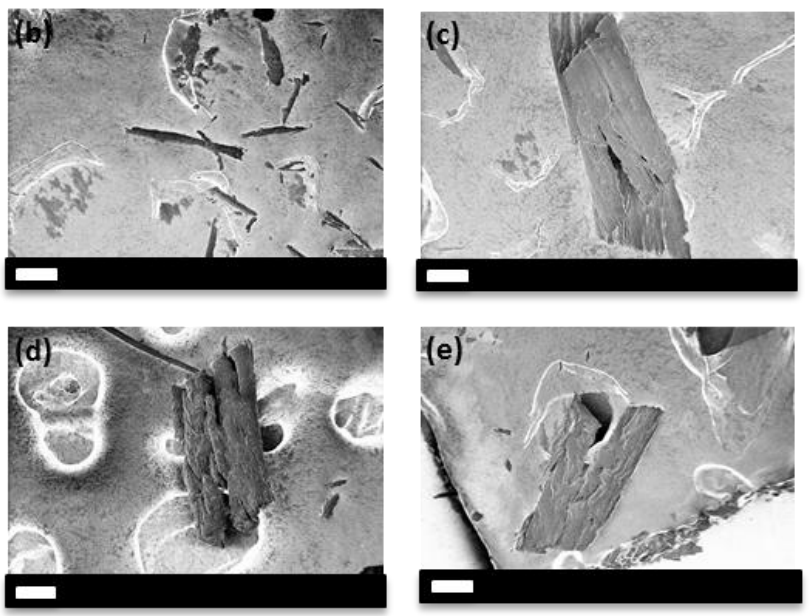

Figure 7: Failure mechanisms in plexiform- $90^{\circ}$ bone (a) heterogenity experienced by the cutting edge; (b)-(e) chip morphology at cutting velocity of $125 \mathrm{~m} / \mathrm{min}$ and FPTs of $1 \mu \mathrm{m}$ , $5 \mu \mathrm{m}, 10 \mu \mathrm{m}$ and $16 \mu \mathrm{m}$, respectively. [Note: continuous chip is formed even at FPT of $5 \mu \mathrm{m}(\mathrm{c})]($ Scale $=100 \mu \mathrm{m})$ 
Figure 8- Single Column
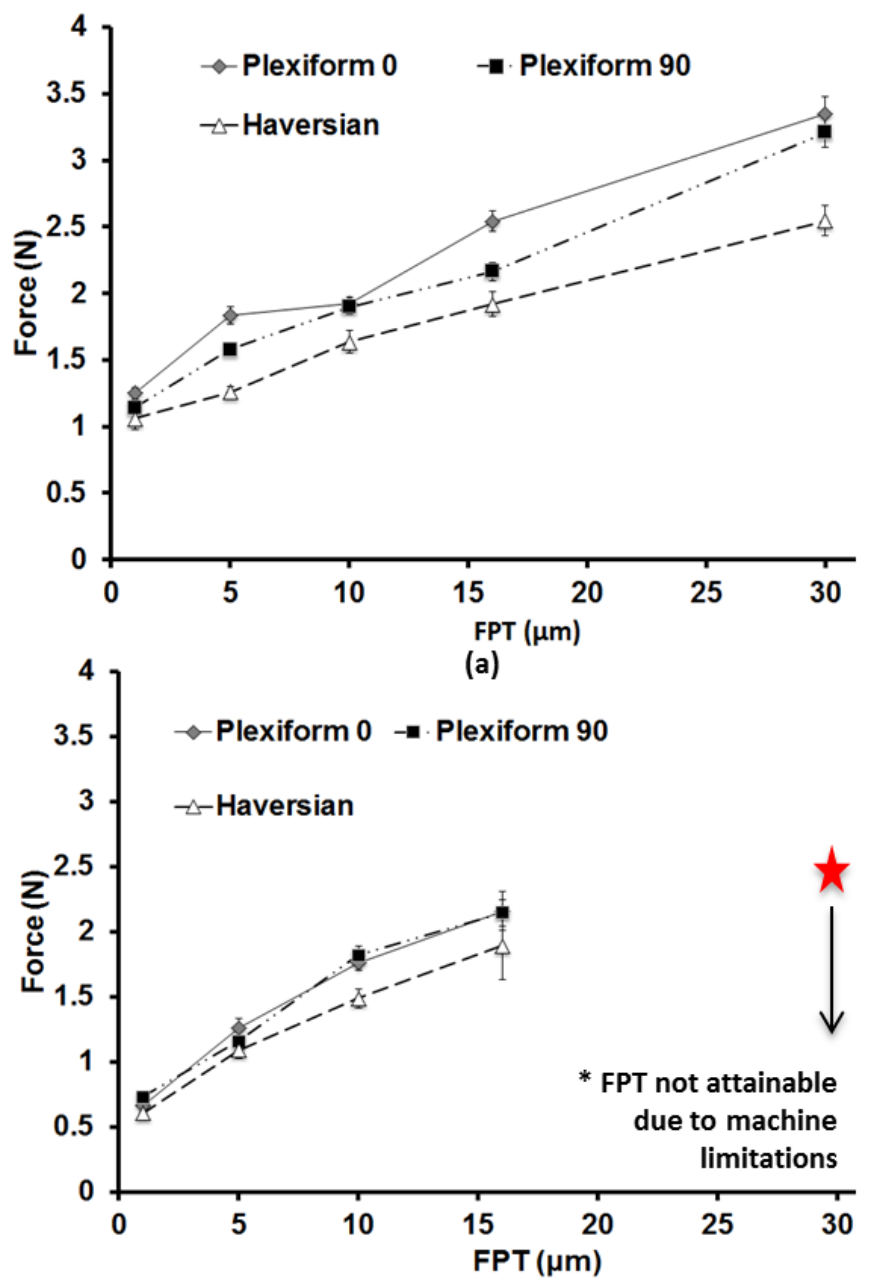

(b)

Figure 8: Resultant cutting force trends (a) cutting velocity 63 $\mathrm{m} / \mathrm{min}$ (b) cutting velocity $125 \mathrm{~m} / \mathrm{min}$. 
Figure 9- Two Column

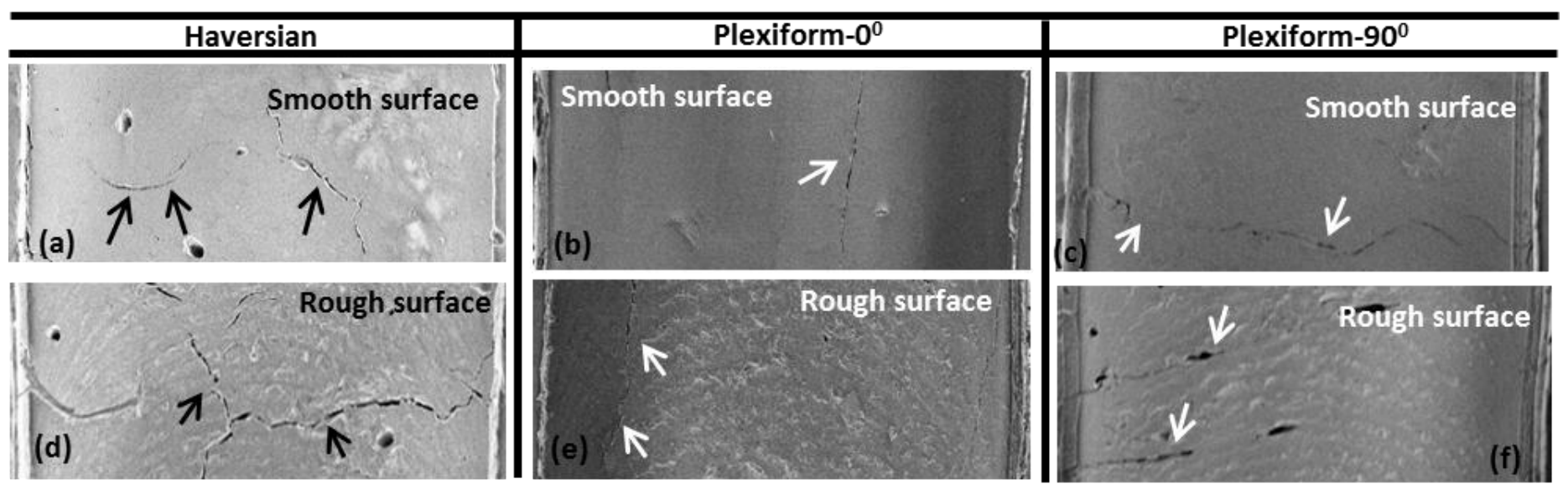

Figure 9: Scanning electron microscopy image of the surface (Cutting velocity of $63 \mathrm{~m} / \mathrm{min}) ;(\mathrm{a}-\mathrm{c}) \mathrm{FPT}=1 \mu \mathrm{m} ;(\mathrm{d}-\mathrm{f}) \mathrm{FPT}=30 \mu \mathrm{m}$ 
Figure 10 - Single Column
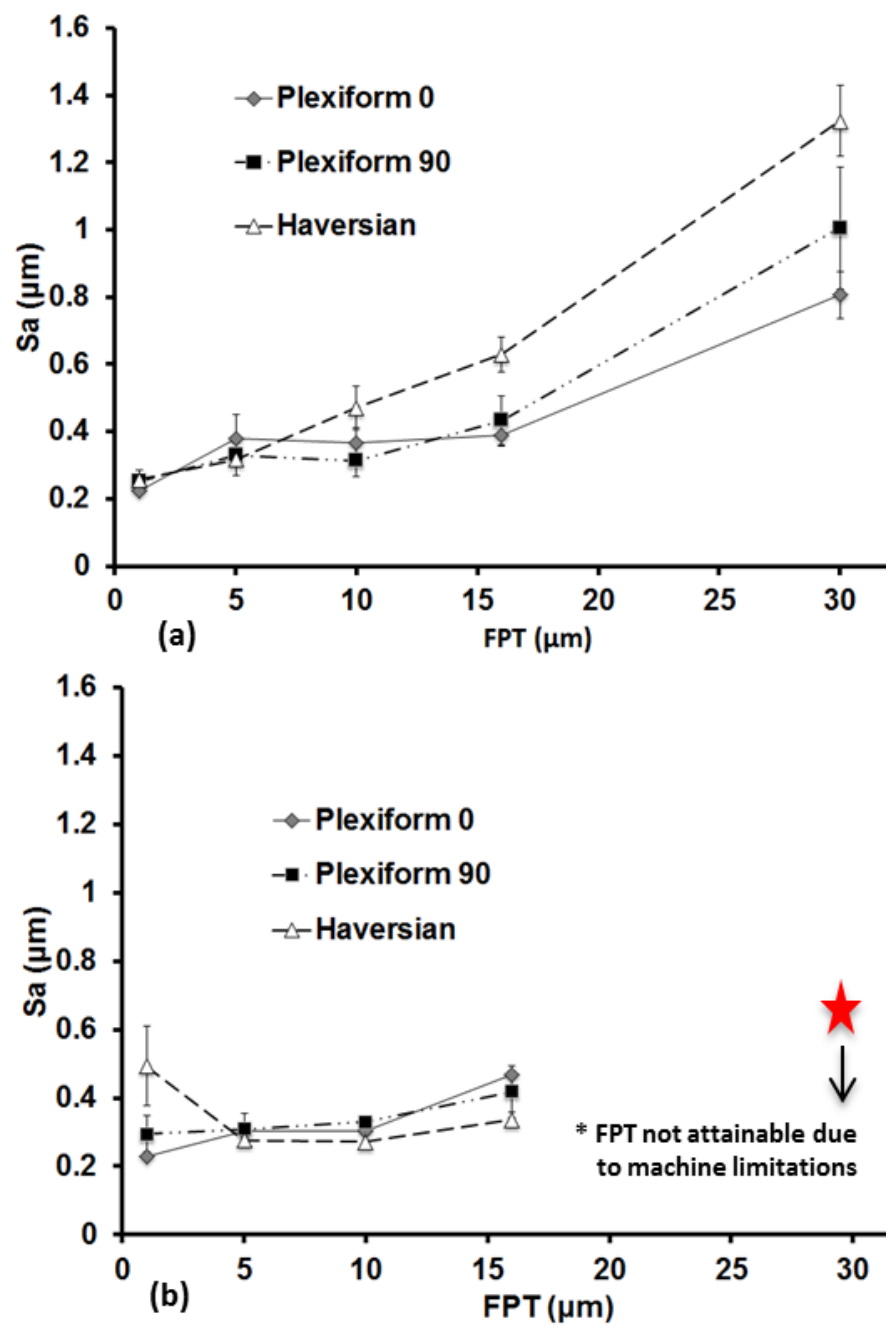

Figure 10: Surface roughness trends (a) cutting velocity 63 $\mathrm{m} / \mathrm{min}$ (b) cutting velocity $125 \mathrm{~m} / \mathrm{min}$. 

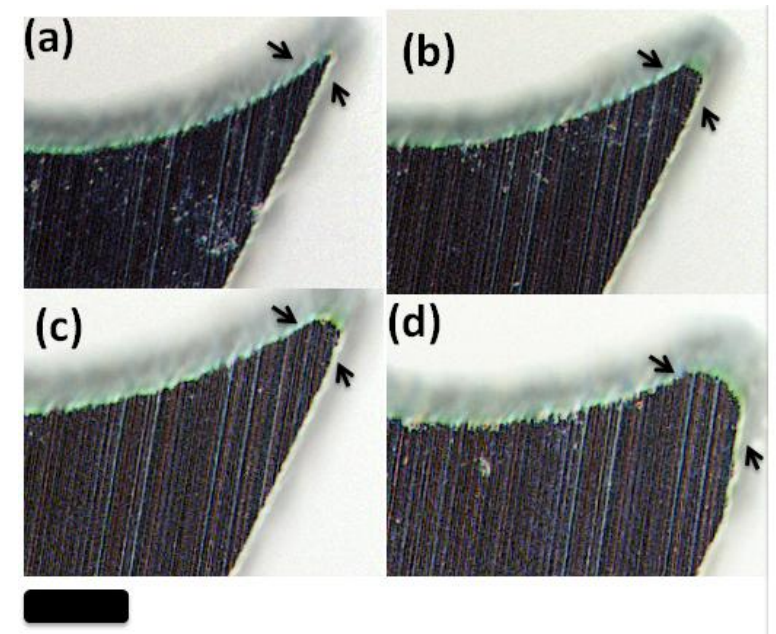

(d)
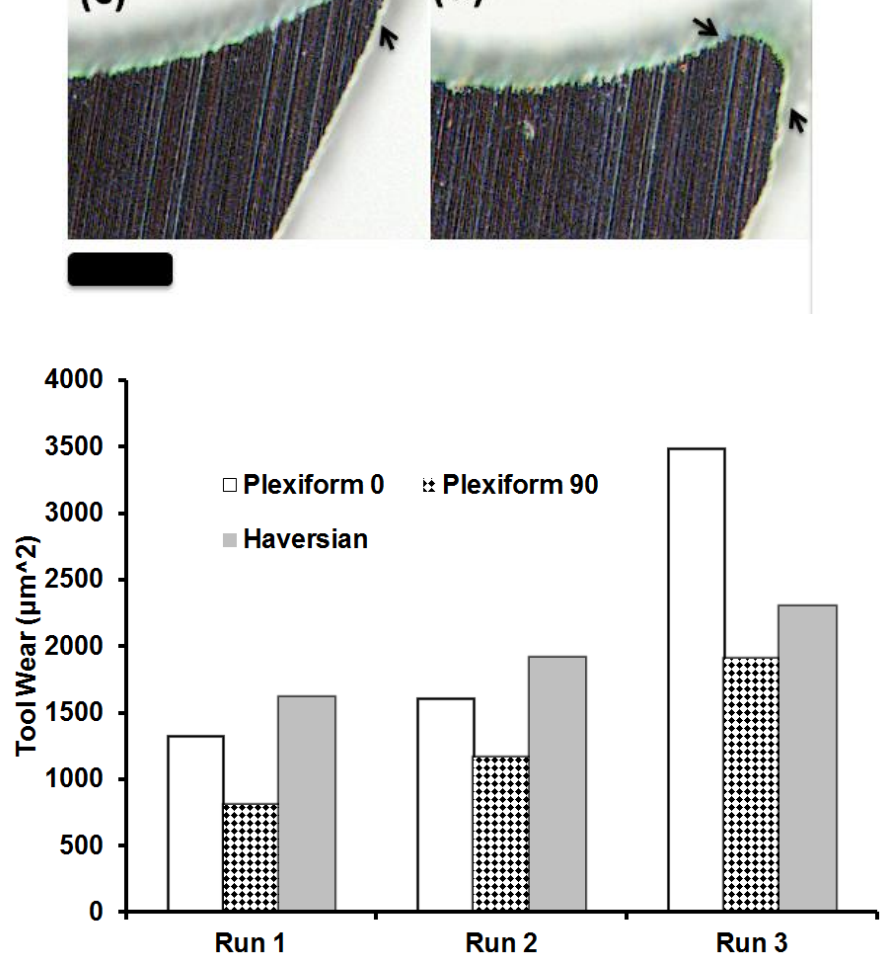

(e)

Figure 11: Tool wear trends for plexiform $-0^{0}$ sample (a) initial tool edge (b) tool edge at the end of Run-1 (c) tool edge at the end of Run-2 (d) tool edge at the end of Run-3 (e) quantitative tool wear data [Scale bar in $(\mathrm{a}-\mathrm{d})=40 \mu \mathrm{m}$ ] 


\section{Table 1 - Single column}

Table 1: Nanoindentation data

\begin{tabular}{|l|c|}
\hline Elastic Modulus & E (GPa) \\
\hline Haversian bone - Osteon & $35.8 \pm 3.50$ \\
\hline Haversian bone - Interstitial Matrix & $36.4 \pm 3.08$ \\
\hline Haversian bone - Cement Line & $22.0 \pm 0.89$ \\
\hline Plexiform Bone - Woven & $38.9 \pm 1.63$ \\
\hline Plexiform Bone - Lamellar & $30.3 \pm 1.89$ \\
\hline Hardness & H (GPa) \\
\hline Haversian bone - Osteon & $1.67 \pm 0.21$ \\
\hline Haversian bone - Interstitial Matrix & $1.73 \pm 0.24$ \\
\hline Haversian bone - Cement Line & $0.81 \pm 0.15$ \\
\hline Plexiform Bone - Woven & $1.91 \pm 0.10$ \\
\hline Plexiform Bone - Lamellar & $1.31 \pm 0.18$ \\
\hline
\end{tabular}


Table 2 - Single column

Table 2: Machining Conditions

\begin{tabular}{|c|c|}
\hline Workpiece & $\begin{array}{l}\text { - } \text { Haversian bone } \\
\text { - } \\
\text { Plexiform- } 0^{0}: \text { Machined parallel to } \\
\text { woven } / \text { lamellar structures } \\
\text { - Plexiform- } 90^{0}: \text { Machined perpendicular } \\
\text { to woven } / \text { lamellar structures } \\
\text { - Workpiece: } 5 \mathrm{~mm} \text { X } 5 \mathrm{~mm} \mathrm{X} 1 \mathrm{~mm}\end{array}$ \\
\hline Tool & $\begin{array}{l}\text { - } 800 \mu \mathrm{m} \text { diameter, single-fluted, tungsten } \\
\text { carbide endmill with } 1 \mu \mathrm{m} \text { edge radius. } \\
\text { (Performance Micro Tool, ET-1-0312-S) }\end{array}$ \\
\hline $\begin{array}{l}\text { Cutting } \\
\text { Speed }\end{array}$ & - $\sim 63 \mathrm{~m} / \mathrm{min}$, and $\sim 125 \mathrm{~m} / \mathrm{min}$ \\
\hline $\begin{array}{l}\text { Depth of } \\
\text { Cut }\end{array}$ & - $\quad 300 \mu \mathrm{m}$ (axial) \\
\hline $\begin{array}{l}\text { Feed-per- } \\
\text { tooth (FPT) }\end{array}$ & - $\quad 1 \mu \mathrm{m}, 5 \mu \mathrm{m}, 10 \mu \mathrm{m}, 16 \mu \mathrm{m}$ and $30 \mu \mathrm{m}$ \\
\hline
\end{tabular}


Graphical Abstract (for review)
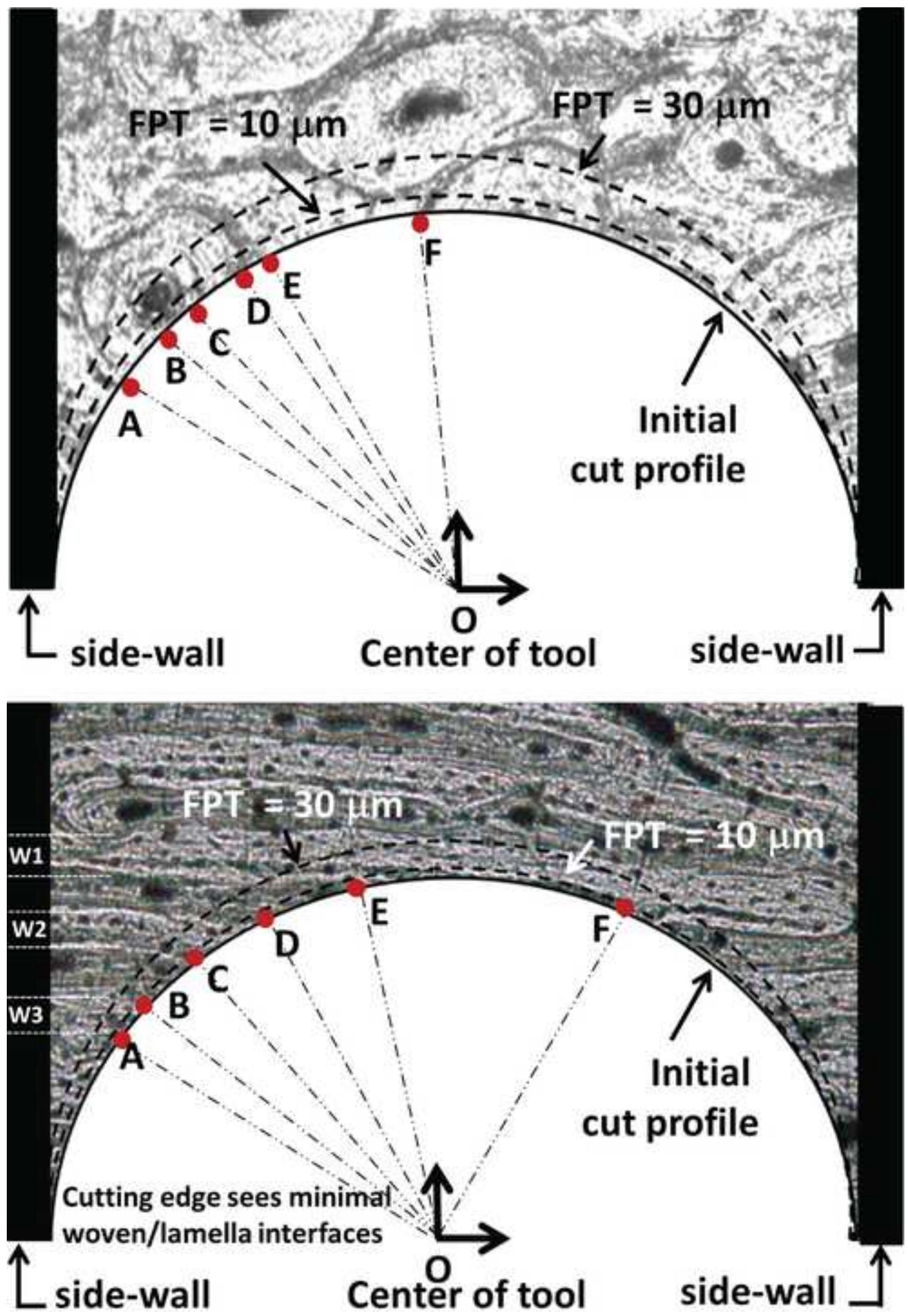